\title{
Conformational Temperature Characterizing the Folding of a Protein
}

\author{
Naoko Nakagawa \\ College of Science, Ibaraki University, Mito, Ibaraki 310-8512, Japan
}

(Dated: March 21, 2007)

\begin{abstract}
The time sequences of the molecular dynamics simulation for the folding process of a protein is analyzed with the inherent structure landscape which focuses on configurational dynamics of the system. Time dependent energy and entropy for inherent structures are introduced and from these quantities a conformational temperature is defined. The conformational temperature follows the time evolution of a slow relaxation process and reaches the bath temperature when the system is equilibrated. We show that the nonequilibrium system is described by two temperatures, one for fast vibration and the other for slow configurational relaxation, while the equilibrium system is by one temperature. The proposed formalism is applicable widely for the systems with many metastable states.

PACS numbers: $05.70 . L n$ 87.15.Cc 05.90.+m
\end{abstract}

Slow relaxations of non-equilibrium systems are a very general process which are typically observed in glasses [1]. For various slow relaxations a major interest is how the relaxation proceeds, and inherent structures, i.e. the local minima of their energy landscape, are powerful to investigate it from the point of energy landscape. Their statistics might provide thermodynamic aspects in system's configuration not only at equilibrium but at nonequilibrium [2 [5].

The folding of proteins provides a nice example of slow relaxation in an out-of-equilibrium complex physical system without homogeneity nor extensivity. It evolves from random coil states to their own native conformation in $\mu$ s or $m s$, while their vibrational dynamics takes place in time scales of the order of $f \mathrm{~s}$ or $p \mathrm{~s}$. Proteins can be investigated in the spirit of glasses by characterizing their inherent structures. We showed that the density of states in the Inherent Structure Landscape (ISL), i.e. the landscape of inherent structure energies, of a model protein depends exponentially on their energy [6], so that the model has some similarities with the simple models of glasses such as spin glasses 7], Lennard-Jones systems [8, 9] and the trap model [10], despite of its nonextensivity and heterogeneity. This letter shows that the ISL is not only useful to study the equilibrium properties of a protein but can also be used in practice to characterize its slow evolution towards the folded configuration by proposing a conformational temperature that follows the time evolution of a slowly relaxing non-equilibrium system.

Model: We consider an off-lattice Gō protein model with a slight frustration 6 . The protein is reduced to its backbone without side chains, each residue being represented by its $C_{\alpha}$ atom. The effective potential between the $C_{\alpha}$ elements in the backbone is designed from the experimentally determined native structure. We study proteinG which has 56 residues [1]. At low temperature, the model protein folds to the native structure, while at high temperature it denatures. Folding-unfolding transi- tion occurs at $T_{f}$, and around $T_{d} \approx 0.4 T_{f}$ a dynamical transition is observed below which the fluctuations of the protein structure are dramatically reduced.

In numerical investigation, molecular dynamics simulations with Langevin equations are applied, where a denatured initial condition is prepared by a simulation at $1.7 T_{f}$ at which the protein is in the random coil state. A sudden cooling to $T<T_{f}$ is applied at $t=0$. For each value of $T, 200$ initial conditions are sampled.

Definitions of quantities: Slow dynamics comes from itinerant transitions among local energy well while the fast dynamics comes from rapid vibrations inside each well. Such slow dynamics can be characterized by "inherent structures" [2], in which the set of conformations belonging to the same basin labeled by the index $\alpha$ are mapped to its local energy minimum with energy $e_{\alpha}$.

Let $w(\Gamma, t, T)$ be the probability density for the system to be at point $\Gamma$, up to $d \Gamma$, in phase space at time $t$ and let $\Gamma_{\alpha}$ be the region corresponding to a basin $\alpha$. The probability weight to observe the basin $\alpha$ is

$$
w_{\alpha}(t, T)=\int_{\Gamma_{\alpha}} w(\Gamma, t, T) d \Gamma
$$

where $\sum_{\alpha} w_{\alpha}(t, T)=1$. The probability weight $w_{\mathrm{v}}^{\alpha}(\Gamma, t, T)$ normalized in the basin $\alpha$ is introduced as

$$
w(\Gamma, t, T)=w_{\alpha}(t, T) \cdot w_{\mathrm{v}}^{\alpha}(\Gamma, t, T) .
$$

To be consistent with Eq.(1), $\int_{\Gamma_{\alpha}} w_{\mathrm{v}}^{\alpha}(\Gamma, t, T) d \Gamma=1$.

For each point $\Gamma$, the value of the energy $e(\Gamma)$ is determined from the model potential. When the point $\Gamma$ belongs to basin $\alpha$, the energy can be rewritten as $e(\Gamma)=e_{\alpha}+\Delta V_{\alpha}(\Gamma)$, where $\Delta V_{\alpha}(\Gamma)$ is the potential at point $\Gamma$ measured from the minimum $e_{\alpha}$. The mean energy $U$ at time $t$ is given by

$$
\begin{aligned}
& U(t, T) \equiv \int e(\Gamma) w(\Gamma, t, T) d \Gamma \\
& \quad=\quad U_{\mathrm{IS}}(t, T)+\sum_{\alpha} U_{\mathrm{v}}^{\alpha}(t, T) w_{\alpha}(t, T) .
\end{aligned}
$$


This expression splits the energy into two components, $U_{\mathrm{IS}}(t, T) \equiv \sum_{\alpha} e_{\alpha} w_{\alpha}(t, T)$ and $U_{\mathrm{v}}^{\alpha}(t, T) \equiv$ $\int_{\Gamma} \Delta V_{\alpha}(\Gamma) w_{\mathrm{v}}^{\alpha}(\Gamma, t, T) d \Gamma$. $U_{\mathrm{IS}}(t, T)$ is the mean inherent structure energy at time $t$ which reflects slow structural changes due to the transition among the basins while $U_{\mathrm{v}}^{\alpha}(t, T)$ is the mean vibrational energy inside basin $\alpha$. This split does not require the separation of time scales between the two modes, but numerical simulations below deal with separated situations.

Since the inherent structure is discrete, the entropy for the inherent structures can be defined as

$$
S_{\mathrm{IS}}(t, T) \equiv-k_{\mathrm{B}} \sum_{\alpha} w_{\alpha}(t, T) \log w_{\alpha}(t, T) .
$$

The total entropy $S(t, T)$ splits into $S_{\mathrm{IS}}(t, T)$ and a vibrational contribution $S_{\mathrm{v}}^{\alpha}(t, T)$ according to

$$
\begin{aligned}
S(t, T) & \equiv-k_{\mathrm{B}} \int w(\Gamma, t, T) \log w(\Gamma, t, T) d \Gamma \\
& =S_{\mathrm{IS}}(t, T)+\sum_{\alpha} S_{\mathrm{v}}^{\alpha}(t, T) w_{\alpha}(t, T) .
\end{aligned}
$$

Here $S_{\mathrm{v}}^{\alpha}(t, T)$ is the vibrational entropy at the basin $\alpha$ such that $S_{\mathrm{v}}^{\alpha}(t, T) \equiv-k_{\mathrm{B}} \int_{\Gamma_{\alpha}} w_{\mathrm{v}}^{\alpha}(\Gamma, t, T) \log w_{\mathrm{v}}^{\alpha}(\Gamma, t, T)$. We add that so-called configuration entropy is not necessarily equal to $S_{\mathrm{IS}}$ even in canonical equilibrium, $S_{\mathrm{IS}, \mathrm{can}}(T) \neq k_{\mathrm{B}} \log \Omega_{\mathrm{IS}}\left(U_{\mathrm{IS}, \mathrm{can}}(T)\right)$, because the value of $e_{\alpha}$ can largely deviate from the mean value $U_{\mathrm{IS}, \mathrm{can}}(T)$ in small systems such as proteins. Here $\Omega_{\mathrm{IS}}\left(e_{\alpha}\right)$ is the density of states for the inherent structures, which can be determined from a numerical simulation in equilibrium situation as shown in [6]. In the present model $\Omega_{\text {IS }}$ is almost an exponential function of $e_{\alpha}$, similarly to simple models of glasses $[7,[8,10]$.

In analogy to the equilibrium case, we define a time-dependent conformational temperature for inherent structures as

$$
\frac{1}{T_{\mathrm{cnf}}(t, T)} \equiv \frac{\partial S_{\mathrm{IS}}(t, T) / \partial T}{\partial U_{\mathrm{IS}}(t, T) / \partial T}
$$

where we expect $T_{\mathrm{cnf}}(t, T) \rightarrow T$ as the relaxation proceeds, and $T_{\mathrm{cnf}}=T$ in thermal equilibrium. Since $S_{\mathrm{IS}}$ and $U_{\mathrm{IS}}$ are a function of $t$ and $T$, one may consider that $\frac{\partial S_{\mathrm{IS}} / \partial t}{\partial U_{\mathrm{IS}} / \partial t}$ can also give a temperature. In fact, the latter corresponds to the internal temperature defined in [4]. We have not adopted this definition because in the present protein system $\frac{\partial S_{\mathrm{IS}} / \partial t}{\partial U_{\mathrm{IS}} / \partial t}$ does not depend on time comparable to the change of the distribution seen in Fig. 2, It is observed to remain in an approximately constant value corresponding to the exponent of $\Omega_{\mathrm{IS}}\left(e_{\alpha}\right)$.

Slow relaxation in Inherent structure landscape: The energetic aspects of the folding process are demonstrated in Fig 1, in which the mean vibrational energy over the phase space is defined as $U_{\mathrm{v}}(t, T) \equiv$ $\sum_{\alpha} U_{\mathrm{v}}^{\alpha}(t, T) w_{\alpha}(t, T)$. As are shown in Fig 1, the mean

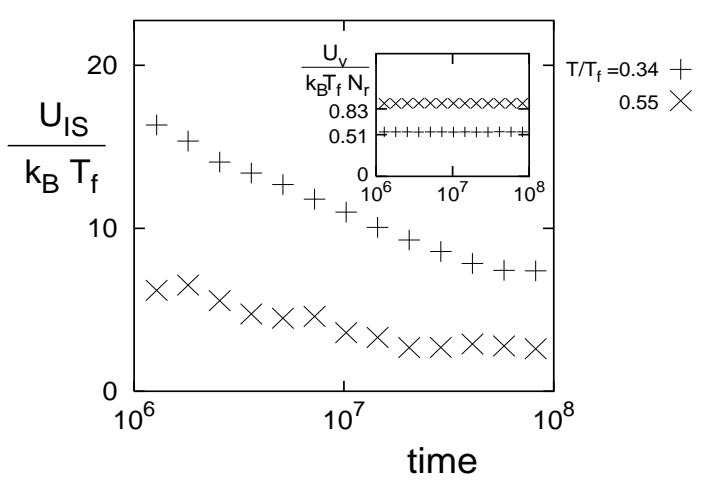

FIG. 1: Time evolution of mean inherent structure energy $U_{\text {IS }}$ and (inset) mean vibrational energy $U_{\mathrm{v}}$. In the case $0.55 T_{f}>T_{d}$, the folding process reaches thermal equilibrium around $t=2 \times 10^{7}$, whereas in $0.34 T_{f}<T_{d}$ it does not within the computation time. $U_{\mathrm{v}}$ is in the equilibrium value which is slightly larger than the harmonic approximation $3 / 2 k_{\mathrm{B}} N_{r} T$ (indicated as 0.51 and 0.83 for $T / T_{f}=0.34$ and 0.55 ). $N_{r}$ is the number of residues.

inherent structure energy $U_{\mathrm{IS}}(t, T)$ relaxes logarithmically in time. Such logarithmic relaxations are typically seen in glassy systems, which suggests intrinsic glassy properties for proteins even above the dynamical transition temperature $T_{d}$.

On the other hand, $U_{\mathrm{v}}(t, T)$ is almost constant, corresponding to its equilibrium value at temperature $T$, although the probability weight $w_{\alpha}(t, T)$ is expected to significantly depend on time as the folding proceeds. This is possible if the vibrational energy $U_{\mathrm{v}}^{\alpha}(t, T)$ is approximately the same for all basins $\alpha$ and the vibrational motions reach equilibrium much faster than the time scale of the folding. The value of $S_{\mathrm{v}}^{\alpha}(t, T)$ is also expected to be equal to its equilibrium value, which could be approximately independent of $\alpha$, similarly to $U_{\mathrm{v}}^{\alpha}(t, T)$.

$S_{\mathrm{IS}}(t, T)$ cannot be accessible to numerical simulation because the number of basins in the whole phase space is too large to determine $w_{\alpha}(t, T)$ for each $\alpha$ and each $t$. Instead of determining $w_{\alpha}(t, T)$ for all basins, let us introduce a probability $P\left(e_{\alpha}, t, T\right)$ to observe the inherent structure energy $e_{\alpha}$,

$$
P\left(e_{\alpha}, t, T\right)=\int d \Gamma w(\Gamma, t, T) \delta\left(e_{\min }(\Gamma)-e_{\alpha}\right) .
$$

Here $e_{\min }(\Gamma)$ is the quenched energy from the point $\Gamma$. If $\Gamma$ belongs to the basin $\alpha, e_{\min }(\Gamma)=e_{\alpha}$.

Since the initial conditions in numerical simulation are randomly selected from the random coil state, let us assume that the weight is approximately the same for basins with the same minimum energy $e_{\alpha}$,

$$
w_{\alpha}(t, T) \simeq P\left(e_{\alpha}, t, T\right) / \Omega_{\mathrm{IS}}\left(e_{\alpha}\right)
$$



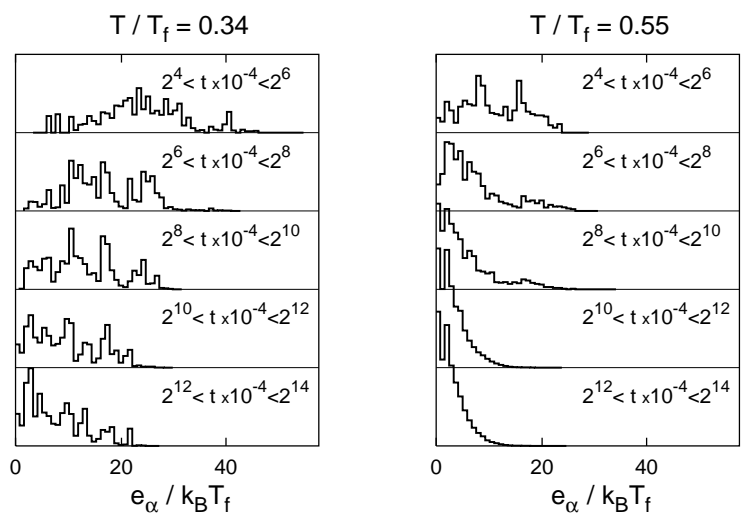

FIG. 2: Time evolution of the probability distribution $P\left(e_{\alpha}, t, T\right)$ for the inherent structure. The horizontal axis is inherent structure energy $e_{\alpha}$ scaled by $k_{\mathrm{B}} T_{f} . T=0.34 T_{f}$ and $0.55 T_{f}$ correspond to $T<T_{d}$ and $T>T_{d}$, respectively. The right bottom figure shows the equilibrium distribution at $T=0.55 T_{f}$. At $t=0$ the protein has been cooled to $T$.

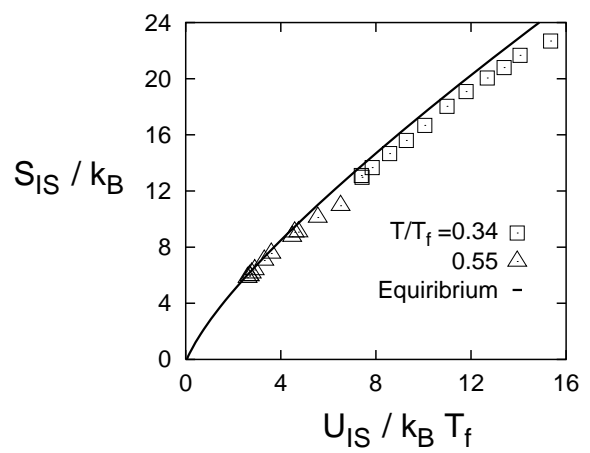

FIG. 3: Time evolution in the plot on $S_{\text {IS }}$ vs $U_{\text {IS }}$ for $t>10^{6}$. $T / T_{f}=0.34$ and 0.55 . The line is the relation between $S_{\text {IS }}$ and $U_{\text {IS }}$ in thermal equilibrium. Higher temperatures corresponds to the right-upper part of the line.

Then,

$$
S_{\mathrm{IS}}(t, T) \simeq-k_{\mathrm{B}} \int d e_{\alpha} P\left(e_{\alpha}, t, T\right) \log \frac{P\left(e_{\alpha}, t, T\right)}{\Omega_{\mathrm{IS}}\left(e_{\alpha}\right)},
$$

which is accessible to numerical simulations.

Examples of the time evolution of $P\left(e_{\alpha}, t, T\right)$ in Fig 2 show that the distribution is strongly dependent on time after the sudden cooling. The energy range that the distribution spans becomes smaller as time proceeds, and the width of the distribution decreases significantly. As is expected $P\left(e_{\alpha}, t, T\right)$ is considerably different from the equilibrium distribution (bottom figure for $T / T_{f}=0.55$ ).

For each time $t$, we can obtain numerically $U_{\text {IS }}(t, T)$ and $S_{\mathrm{IS}}(t, T)$ following the definition (9). If we plot $S_{\mathrm{IS}}(t, T)$ as a function of $U_{\mathrm{IS}}(t, T)$ for various $t$, we get the time evolution for the relaxation process shown in Fig 3. For comparison we plot the line corresponding to thermal equilibrium cases over a wide range of $T$. This

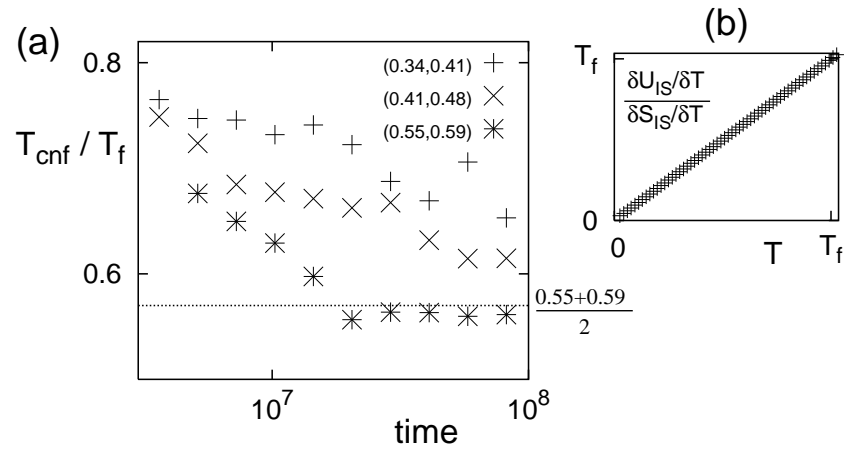

FIG. 4: (a) Time evolution of $T_{\mathrm{cnf}}\left(t,\left(T_{1}+T_{2}\right) / 2\right)$ in eq. (6) estimated by $\left\{S_{\mathrm{IS}}\left(t, T_{2}\right)-S_{\mathrm{IS}}\left(t, T_{1}\right)\right\} /\left(T_{2}-T_{1}\right)$ and $\left\{U_{\mathrm{IS}}\left(t, T_{2}\right)-U_{\mathrm{IS}}\left(t, T_{1}\right)\right\} /\left(T_{2}-T_{1}\right)$. Here $\left(T_{1}, T_{2}\right)=$ $\left(0.34 T_{f}, 0.41 T_{f}\right),\left(0.41 T_{f}, 0.48 T_{f}\right)$ and $\left(0.55 T_{f}, 0.59 T_{f}\right)$. The dotted horizontal line is $\left(T_{1}+T_{2}\right) / 2$ for $\left(0.55 T_{f}, 0.59 T_{f}\right)$, which is expected to be a convergence line of $T_{\operatorname{cnf}}\left(t, 0.57 T_{f}\right)$. (b) Confirmation of thermodynamic relation, $T_{\mathrm{cnf}}=T$ in thermal equilibrium condition.

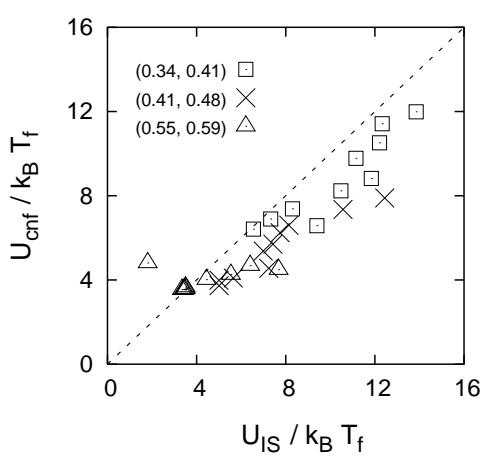

FIG. 5: (a) $U_{\text {cnf }}(t, T)$ vs $U_{\text {IS }}(t, T)$. Here $T=\left(T_{1}+\right.$ $\left.T_{2}\right) / 2$ with $\left(T_{1}, T_{2}\right)=\left(0.34 T_{f}, 0.41 T_{f}\right),\left(0.41 T_{f}, 0.48 T_{f}\right)$ and $\left(0.55 T_{f}, 0.59 T_{f}\right)$.

comparison makes it clear that the time evolution in a fixed cooling temperature $T$ proceeds in parallel to the line for thermal equilibrium from high to low temperatures. Let us study this evolution by the conformational temperature $T_{\mathrm{cnf}}(t, T)$ defined in eq. (6). In equilibrium, $S_{\text {IS }}$ and $U_{\text {IS }}$ work well to determine the temperature of the system as is demonstrated in Fig. प(b), where $T_{\mathrm{cnf}}$ for the canonical ensemble with $\Omega_{\mathrm{IS}}\left(e_{\alpha}\right)$ has the expected property, $T_{\mathrm{cnf}}=T$. In the relaxation process, $T_{\mathrm{cnf}}$ decreases with time from a higher temperature. When $T$ is sufficiently high, we can observe that $T_{\text {cnf }}$ converges to a value corresponding to $T$ after the relaxation to equilibrium ( $T \simeq 0.55 T_{f}$ in Fig. $\left.4(\mathrm{a})\right)$. Thus the features observed in Fig. 2 or Fig. 3 in the relaxation process are reflected in $T_{\text {cnf }}(t, T)$.

The value of $T_{\mathrm{cnf}}(t, T)$ is expected to give an approximation of $P\left(e_{\alpha}, t, T\right)$ to an equilibrium distribution at 
the temperature $T_{\mathrm{cnf}}$. To check it, we compare the approximated mean IS-energy $U_{\text {cnf }}(t, T)$ with $U_{\text {IS }}(t, T)$ in Fig. [5 where

$$
U_{\mathrm{cnf}}(t, T) \equiv \int d e_{\alpha} \frac{e_{\alpha} \Omega_{\mathrm{IS}}\left(e_{\alpha}\right)}{Z_{\mathrm{IS}}\left(T_{\mathrm{cnf}}(t, T)\right)} \exp \left(\frac{-e_{\alpha}}{k_{B} T_{\mathrm{cnf}}(t, T)}\right) .
$$

The figure displays the tendency $U_{\mathrm{cnf}}(t, T) \simeq U_{\mathrm{IS}}(t, T)$, which suggests that $T_{\mathrm{cnf}}$ can give a first approximation for the distribution $P\left(e_{\alpha}, t, T\right)$. The deviation of the points from the line in Fig. 5 indicates that the states on the way of folding do not coincide strictly with any equilibrium state. Correspondingly, the points in Fig. 3 deviates slightly from the equilibrium line.

Discussion: We have introduced time dependent energies and entropies for inherent structures to analyze the slow folding process of a model protein. After a sudden cooling, we found that the probability distribution of the inherent structures is widely spread in the early stage and becomes narrower as time proceeds. This evolution is successfully characterized by the time evolution of the conformational temperature Eq.(6), as shown in Fig 4(a).

Although the approximation of the distribution by $T_{\mathrm{cnf}}$ is not complete, we note that even this first approximation is not obtained if we treat the total probability distribution without separating the inherent structure modes and the vibrational modes. As argued in Fig 1 the vibrational modes are reaching local equilibrium in the early stage. Thus there is a temperature difference between the fast dynamics and the slow dynamics. Thermodynamic frameworks including two temperatures could be proposed [3]. It might be interesting to study various slow dynamics adopting such two temperatures.

Sciortino and Tartaglia [4] introduced an internal temperature for glasses as a differential of two temporal states characterized by the inherent structure energy and configuration entropy. The internal temperature appears to approximate temporal distribution $P\left(e_{\alpha}, t, T\right)$ in some glasses [5] although we observe that it does not in the protein model. From these studies, we expect that internal temperature coincides with confromational temperature in glassy systems in contrast with their disagreement in the protein model. This difference might reprenst a defference in a basic property of the system, i.e., glasses are homogeneous and extensive but single proteins are heterogeneous and nonextensive. The measurement of effective temperature in protein models, which is defined by the violation of fluctuation-dissipation relation [12, 13] and has known to coincide with the internal temperature in a Lenard-Jones or a spin glass [4, 5], is a future work to be studied. This is not a simple task because the effective temperature could be observable-dependent [10] and the intensive variable for the protein models is not obvious.
Finally we would like to point out that this study emphasizes the interest of the Inherent Structure Landscape for protein. We showed earlier [6] that, contrary to the free-energy landscape which is only a useful concept, the density of states of the ISL can be obtained in practice for a protein model, and that it characterizes the equilibrium properties of the protein to a good accuracy. Here we show that the ISL is not only useful in equilibrium, but can be used to characterize the slow relaxation with a time-dependent conformational temperature. The assumption used in this letter, that $U_{\mathrm{v}}^{\alpha}$ and $S_{\mathrm{v}}^{\alpha}$ does not depend on $\alpha$, appears to be good for the protein case. It can be relaxed for more complex systems [14].

Note: Since the present model possesses at least two funnel which is well separated from the others, we choose the relaxation process to a funnel of which global minimum energy state corresponds to a misfolded state. Its minimum energy state resembles the native state but a $\alpha$-helix is bent compared to the native state. In this funnel, relaxation proceeds in a time scale accessible to numerical simulations if the temperature is not too low.

Acknowledgement: N.N. thanks M. Peyrard for useful discussion and critical reading of the manuscript. She also thanks MEXT KAKENHI (No. 16740217) for support.

[1] Slow relaxations and nonequilibrium dynamics in condensed matter, J.L. Barrat et al Eds., EDP Sciences; Springer-Verlag (2003).

[2] F. H. Stillinger and T. A. Weber, Phys. Rev. A, 25 (1982) 978.

[3] Th. M. Nieuwenhuizen, Phys. Rev. Lett. 80 (1998) 5580.

[4] F. Sciortino and P. Tartaglia, Phys. Rev. Lett. 86 (2001) 107.

[5] A. Crissanti and F. Ritort, J. Phys: Condens. Matter 14 (2002) 1381.

[6] N. Nakagawa and M. Peyrard, Proc. Nat. Acad. Sci., 103 (2006) 5279.

[7] A. J. Bray and M. A. Moore, J. Phys. C: Solid State Phys. 14 (1981) 1313.

[8] J.P.K. Doye and D.J. Wales, J. Chem. Phys. 102 (1995) 9659.

[9] F. Sciortino, W. Kob, and P. Tartaglia, Phys. Rev. Lett. 83 (1999) 3214.

[10] S. Fielding and P. Sollich, Phys. Rev. Lett. 88 (2002) 05603.

[11] H. M. Berman et al, Nucleic Acids Research, 28 (2000) 235.

[12] L. F. Cugliandolo and J. Kurchan, J. Phys. A, 27 (1994) 5749.

[13] L. F. Cugliandolo, J. Kurchan and L. Peliti, Phys.Rev.E, 55 (1997) 3898.

[14] N. Nakagawa and M. Peyrard, in preparation. 